\title{
Review of Effects of Physical Activity on Strength, Balance, Mobility and ADL Performance in Elderly Subjects with Dementia
}

\author{
Christiaan G. Blankevoort $^{\mathrm{a}} \quad$ Marieke J.G. van Heuvelen ${ }^{\mathrm{a}} \quad$ Froukje Boersma $^{\mathrm{b}}$ \\ Helga Luning $^{a}$ Jeltsje de Jong ${ }^{c}$ Erik J.A. Scherder ${ }^{a, d}$ \\ a Institute of Human Movement Sciences and ${ }^{b}$ Department of General Medicine and Geriatrics, University \\ Medical Center Groningen, Groningen, ' Nursing Home Vierackers, Mental Health Center Drenthe, Assen, \\ and ${ }^{\mathrm{d}}$ Department of Clinical Neuropsychology, VU University Amsterdam, Amsterdam, The Netherlands
}

\section{Key Words}

Physical activity $\cdot$ Alzheimer's disease $\cdot$ Dementia $\cdot$

Activities of daily living • Progressive resistance training •

Systematic review $\cdot$ Exercise

\begin{abstract}
Background/Aims: Elderly individuals with dementia are vulnerable for a decline in physical functioning and basic activities of daily living (BADL) which can lead to a decline in autonomy and participation. This study reviews the effect of physical activity on physical functioning and BADL in elderly subjects with dementia. Methods: A systematic search of the literature was performed. Key words related to the elderly, dementia, exercise interventions and physical outcome measures were used. Results: Sixteen studies were included. It was found that physical activity was beneficial in all stages of dementia. Multicomponent interventions (e.g. a combination of endurance, strength and balance) led to larger improvements in gait speed, functional mobility and balance, compared to progressive resistance training alone. BADL and endurance improved but were only assessed in multicomponent interventions. Lower-limb strength improved equally in multicomponent interventions and progressive resistance training. Conclusion: Multicomponent interventions can improve physical functioning and BADL in elderly
\end{abstract}

subjects regardless of the stage of dementia. The best results were obtained in the interventions with the largest training volume. However, the small number of high-quality studies, and heterogeneity of the participants and interventions prevent us from drawing firm conclusions. Recommendations are given with respect to methodological issues, further research and practical guidelines.

Copyright $\odot 2010$ S. Karger AG, Basel

\section{Introduction}

At this moment there are 24.3 million people with dementia of multiple etiologies, e.g. Alzheimer's disease, vascular dementia or frontotemporal dementia, worldwide. It is estimated that this number will double every 20 years to 81.1 million in 2040 [1]. Since there is no cure for dementia [2], the increase in the number of people with dementia will have a great impact on our national health care systems [3]. In addition to disturbances in cognition and behavior [4], dementia leads to a deterioration in the performance of activities of daily living (ADL) $[5,6]$. ADL can be divided into instrumental ADL, which include activities like light housework, preparing meals or taking medication, and basic ADL (BADL), such as bathing, eating and dressing [7]. The deterioration in the performance

\section{KARGER}

Fax +4161306 1234 E-Mail karger@karger.ch www.karger.com
(C) 2010 S. Karger AG, Basel

$1420-8008 / 10 / 0305-0392 \$ 26.00 / 0$

Accessible online at:

www.karger.com/dem
Christiaan G. Blankevoort, MSc

Center for Human Movement Sciences

University Medical Center Groningen, PO Box 196

NL-9700 AD Groningen (The Netherlands)

Tel. +31 50363 2611, Fax +31 50363 3150, E-Mail c.g.blankevoort@ @ed.umcg.nl 
of BADL, especially, leads to a decline in autonomy [7] and is, consequently, an important cause of institutionalization $[6,8]$. Some argue that the decline in BADL is a greater burden for caregivers than the decline in cognition [9]. Moreover, the decline in BADL in particular accelerates in moderate dementia $[10,11]$. Therefore, it is important to improve or stabilize the ability to perform BADL.

The model of the International Classification of Functioning, Disability and Health (fig. 1) [12] can describe the consequences of dementia that eventually lead to deterioration in BADL and loss of autonomy. In the context of this review, dementia (health condition) has a negative influence on mobility, endurance, lower-extremity strength and balance (body functions and body structures). Those body functions are important for BADL functioning (activity). Depending on the quality of the BADL performance, patients are less or more restricted in their participation (participation). Improvement in the other components is a prerequisite for improvement in participation. Unfortunately, dementia cannot be cured, but the consequences may be influenced. The body functions of people (e.g. mobility, lower-extremity strength, balance and walking endurance) are highly trainable in cognitively intact older adults $[13,14]$ and can lead to an improvement in ADL [15]. By training physical components underlying $\mathrm{ADL}$, or by a direct influence of exercise on ADL, healthy elderly subjects can stabilize or improve their ADL score [15].

The next question that arises is whether training of mobility, lower-extremity strength, balance and walking endurance is also effective, directly or indirectly, in improving ADL performance in older persons with dementia. Therefore, the main goal of this review is to investigate whether physical activity can improve mobility, lower-extremity strength, balance, walking endurance and BADL in elderly individuals with dementia. To formulate recommendations for the most effective training program for elderly subjects with dementia, we will address specific training parameters such as duration, frequency, intensity and participation rate.

\section{Methods}

\section{Search Strategy}

Between September 2009 and March 2010, Embase, Pubmed, Web of Science (ISI), Psychinfo, CINAHL and Biological Abstracts (ISI) were searched for publications on physical activity provided to elderly subjects with dementia. Key words used in the computerized search included terms from Medical Subject Headings and Embase thesaurus as well as free text terms. For searches

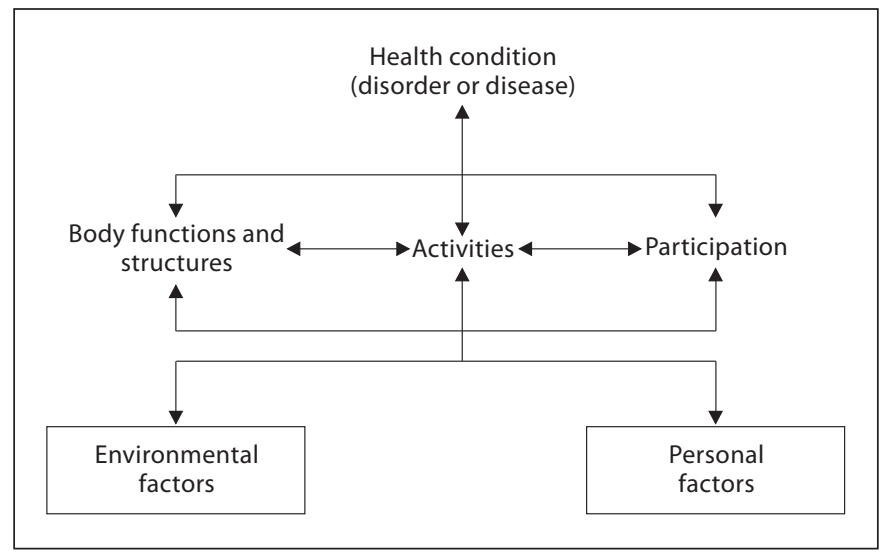

Fig. 1. The International Classification of Functioning, Disability and Health model [12].

in the Medical Subject Headings database, we used the following terms: motor activity, exercise, physical activity, dementia, aged, musculoskeletal physiological phenomena, physical fitness, activities of daily living; for the Emtree database search, we used motor activity, exercise, physical activity, dementia, aged, musculoskeletal function, fitness, strength, endurance, aerobic capacity, daily life activity. In both searches the following free text terms were added: physical training, mobility, frail, Alzheimer, balance, functional performance, functional limitations.

Key words standing for exercise (motor activity, exercise, physical activity, physical training) were combined (with 'and') with terms that expressed the population (dementia, aged, frail, Alzheimer), which were combined (with 'and') for our outcome measures (musculoskeletal physiological phenomena, musculoskeletal function, strength, mobility, balance, endurance, aerobic capacity, functional performance, functional limitation, physical fitness, activities of daily living, ADL). In addition, the reference lists of all the selected studies were thoroughly screened for additional studies.

\section{Inclusion Criteria}

Studies were included if they met the following 5 criteria: (1) participants had dementia of any etiology; (2) participants were on average older than 70, to prevent incorporation of early-onset dementia; (3) solely the effects of physical activity were investigated; (4) the study included outcome measures related to mobility, endurance, lower-extremity strength, balance or ADL performance, and (5) studies were written in English, Dutch, French or German.

\section{Analyses of Published Studies}

A first selection was made on title level and a second after reading the abstracts. In both steps, a conservative approach was used, which means that if there was any doubt, the abstract or full text was screened. Two reviewers (C.G.B. and M.v.H.) performed these steps independently. The process is presented in the flow chart (fig. 2). If both reviewers thought a study was irrelevant, this study was excluded. The full-text analysis of the different studies was performed by one reviewer (C.G.B.). 


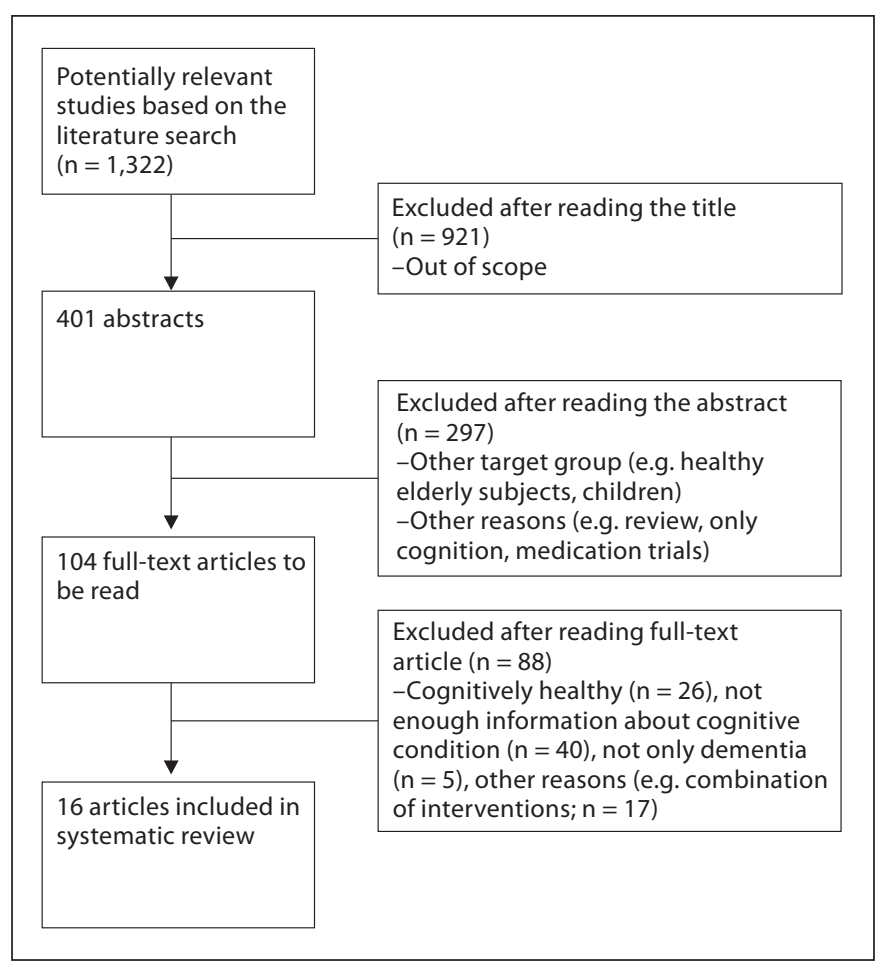

Fig. 2. Flowchart of literature search and study selection.

\section{Methodological Quality}

After this initial process, the same reviewer scored the methodological quality of randomized and nonrandomized trials using the Downs and Black (DB) checklist [16]. From this validated and reliable checklist, 26 questions were used in 4 different domains: reporting (score $0-11$ ), external validity (score $0-3$ ), internal validity - bias, which might indicate a difference between the groups (score $0-7$ ), and internal validity - confounding, which might indicate the existence of a third variable (score $0-6$ ). The maximum score is 27; a higher score indicates a greater methodological quality [16].

In addition, we used the list provided by Sackett et al. [17]. This list provides 5 different levels of evidence ranging from 1 to 5 where 1 is the best score available and 5 is the lowest score. Level 1A: systematic reviews of randomized controlled trials (RCTs); 1B: individual RCTs with narrow confidence intervals and double blind; 2A: systematic reviews of cohort studies; 2B: individual cohort studies and low-quality RCTs (e.g. single-blind RCTs, quasi experimental design or low sample sizes); $3 \mathrm{~A}$ : systematic reviews of case-control studies; 3B: case-control studies; 4: case series and poor-quality cohort and case-control studies; 5 : expert opinion.

In this field of research, it is impossible to perform a doubleblind intervention; therefore, the highest level of evidence that can be reached is $2 \mathrm{~B}$.

\section{Data Extraction}

From the studies selected for full analysis, the following data were extracted: authors, publication year, study design, study analysis (intention-to-treat or per-protocol), sample size, the rate of enrollment, the number of dropouts, the participation rate, percentage of females, mean age, mean cognitive function, data regarding the type of intervention (progressive resistance training, endurance training or a combination of strength, endurance and balance training), duration (weeks/months), frequency (number of sessions per week), length of sessions (minutes) and the training intensity (light, moderate or high).

The rate of enrollment is the percentage of participants who met the inclusion and exclusion criteria and participated in the study. Dropouts were participants who were included in the intervention but who left the program, due to hospitalization, sickness, death and refusal. The participation rate is calculated based on the attendance of participants who participated in the study.

\section{Statistical Analyses}

Cohen's d effect sizes (ESs) [18] were calculated. If an ES was calculated from an RCT, the following formula was used:

$$
\begin{aligned}
& \mathrm{d}=\left[\left(\text { post }_{\exp }-\text { pre }_{\exp }\right)-\left(\text { post }_{\text {cont }}-\text { pre }_{\text {cont }}\right)\right] / \\
& \operatorname{Sqrt}\left[\left(\left[\mathrm{s}^{2} \text { pre }_{\exp }\left(\mathrm{n}_{\exp }\right)+\mathrm{s}^{2} \text { pre }_{\text {cont }}\left(\mathrm{n}_{\text {cont }}\right)\right] /\left[\mathrm{n}_{\exp }+\mathrm{n}_{\text {cont }}\right]\right)+\right. \\
& \left.\left.\left(\left[\mathrm{s}^{2} \text { post }_{\text {exp }}\left(\mathrm{n}_{\exp }\right)+\mathrm{s}_{\text {post }} \text { cont }_{\text {cont }}\right)\right] /\left[\mathrm{n}_{\exp }+\mathrm{n}_{\text {cont }}\right]\right) / 2\right][18,19] \text {. }
\end{aligned}
$$

If a control group was not present, the following formula was used [20]:

$$
\begin{aligned}
& \mathrm{d}=\operatorname{mean}_{\text {post }}-\text { mean }_{\text {pre }} / \operatorname{Sqrt}\left[\left(\mathrm{s}^{2}{ }_{\text {pre }}\left[\mathrm{n}_{\text {pre }}\right]+\right.\right. \\
& \left.\left.\mathrm{s}^{2}{ }_{\text {post }}\left[\mathrm{n}_{\text {post }}\right]\right) /\left(\mathrm{n}_{\text {pre }}+\mathrm{n}_{\text {post }}\right)\right] .
\end{aligned}
$$

If the ES had to be calculated from an F value, the following formula was used [20]:

$$
\begin{aligned}
& \mathrm{d}=\operatorname{Sqrt}\left[\mathrm { F } \left(\left[\left(\mathrm{n}_{\exp }+\mathrm{n}_{\text {cont }}\right) /\left(\mathrm{n}_{\exp } \cdot \mathrm{n}_{\text {cont }}\right)\right] \cdot\right.\right. \\
& \left.\left.\left[\left(\mathrm{n}_{\exp }+\mathrm{n}_{\text {cont }}\right) /\left(\mathrm{n}_{\exp }+\mathrm{n}_{\text {cont }}-2\right)\right]\right)\right] .
\end{aligned}
$$

The overall ES is calculated as the mean of individual ESs weighted for the sample size

An ES of 0.2 was taken to be indicative of a small, 0.5 of a medium and 0.8 of a large ES [21]. If the ES could not be calculated due to a lack of information in the article, the level of significance (p) is provided.

\section{Results}

\section{Study Characteristics}

The literature search and hand search of references revealed 1,322 potentially relevant studies. After the initial screening on title and abstract level, 104 full-text articles were retrieved for further analyses. Finally, a total of 16 studies met the inclusion criteria and were incorporated in this review (fig. 2) [22-37]. Details of these studies, the results, ESs and level of significance, are presented in table 1. Information about the rate of enrollment, the number of dropouts and the participation rate is presented in table 2. In total, there were 10 RCTs of level $2 \mathrm{~B}$ evidence with an average score on the DB questionnaire of 15.2 (range 11-23). These studies scored $56.3 \%$ of the maxi- 
Table 1. Study characteristics and outcome measures

\begin{tabular}{|c|c|c|c|c|c|c|c|c|c|c|c|}
\hline Author & Design & $\begin{array}{l}\mathrm{n} \text { and } \\
\% ?\end{array}$ & $\begin{array}{l}\text { Age } \\
\text { years }^{\mathrm{a}}\end{array}$ & MMSE $^{\mathrm{a}}$ & Intervention & $\begin{array}{l}\text { Functional } \\
\text { outcome } \\
\text { measure }\end{array}$ & $\begin{array}{l}\mathrm{p} \\
\text { value }\end{array}$ & $\begin{array}{l}\text { Cohens' } \\
\text { d }\end{array}$ & Analysis & $\begin{array}{l}\text { Sackett } \\
\text { level of } \\
\text { evidence }\end{array}$ & $\begin{array}{l}\text { DB } \\
\text { checklist }\end{array}$ \\
\hline $\begin{array}{l}\text { Aman and } \\
\text { Thomas } \\
{[22], 2009}\end{array}$ & $\begin{array}{l}\text { Case } \\
\text { series }\end{array}$ & $\begin{array}{l}\text { EG } 40 \\
77.5 \% \\
\text { CG } 10 \\
70 \%\end{array}$ & $\begin{array}{l}78.8 \\
(8.7) \\
81.1 \\
(13.4)\end{array}$ & $\begin{array}{l}1.4^{\mathrm{b}} \\
(1.8) \\
1.7^{\mathrm{b}} \\
(2.9)\end{array}$ & $\begin{array}{l}3 \text { weeks, } 9 \text { sessions of } 30 \mathrm{~min} \text {, } \\
50 \% \text { aerobic and } 50 \% \text { resistance } \\
\text { exercises; } 270 \mathrm{~min} \text { in total }\end{array}$ & $\begin{array}{l}\text { Gait speed } \\
\text { ADL }\end{array}$ & $\begin{array}{l}0.001 \\
0.108\end{array}$ & $\begin{array}{l}0.50 \\
0.11\end{array}$ & $\begin{array}{l}\text { Intention- } \\
\text { to-treat } \\
\text { and per- } \\
\text { protocol }\end{array}$ & 4 & $\begin{array}{l}\text { Report. } 8 \\
\text { Ext. val. } 4 \\
\text { Int. val. bias } 3 \\
\text { Int. val. conf. } 4 \\
\text { Total } 19\end{array}$ \\
\hline $\begin{array}{l}\text { Arkin } \\
{[33], 2003}\end{array}$ & $\begin{array}{l}\text { Case } \\
\text { series }\end{array}$ & $\begin{array}{l}24 \\
66.6 \%\end{array}$ & $\begin{array}{l}78.8 \\
(8.04)\end{array}$ & n.a. & $\begin{array}{l}2-8 \text { semesters, } 10 \text { weeks each } \\
\text { semester, twice a week, } \\
40-60 \text { min; strength, balance } \\
\text { and aerobic exercises; } \\
1,600-9,600 \text { min in total }\end{array}$ & $\begin{array}{l}\text { 6-min walk test } \\
\text { Lower-extremity } \\
\text { strength }\end{array}$ & $\begin{array}{l}<0.001 \\
<0.001\end{array}$ & $\begin{array}{l}3.79 \\
2.73\end{array}$ & $\begin{array}{l}\text { Per- } \\
\text { protocol }\end{array}$ & 4 & $\begin{array}{l}\text { Report. } 3 \\
\text { Ext. val. } 0 \\
\text { Int. val. bias } 1 \\
\text { Int. val. conf. } 0 \\
\text { Total } 4\end{array}$ \\
\hline $\begin{array}{l}\text { Binder } \\
{[23], 1995}\end{array}$ & $\begin{array}{l}\text { Case } \\
\text { series }\end{array}$ & $\begin{array}{l}14 \\
50 \%\end{array}$ & $\begin{array}{l}88.7 \\
(6.9)\end{array}$ & $\begin{array}{l}14.0 \\
(8.0)\end{array}$ & $\begin{array}{l}8 \text { weeks, } 3 \text { times a week, } \\
50-60 \text { min of strength, flexibility, } \\
\text { speed of movement exercises; } \\
1,200-1,440 \text { min in total }\end{array}$ & $\begin{array}{l}\text { Gait speed } \\
\text { Sit-to-stand test } \\
\text { Balance }\end{array}$ & $\begin{array}{r}>0.05 \\
>0.05 \\
0.002\end{array}$ & $\begin{array}{l}0.29 \\
0.34 \\
0.53\end{array}$ & $\begin{array}{l}\text { Per- } \\
\text { protocol }\end{array}$ & 4 & $\begin{array}{l}\text { Report. } 5 \\
\text { Ext. val. } 1 \\
\text { Int. val. bias } 3 \\
\text { Int. val. conf. } 0 \\
\text { Total } 9\end{array}$ \\
\hline $\begin{array}{l}\text { Christa- } \\
\text { foletti } \\
\text { et al. } \\
{[24], 2008}\end{array}$ & $\mathrm{RCT}$ & $\begin{array}{l}\text { EG } 17 \\
71 \% \\
\text { CG } 20 \\
70 \%\end{array}$ & $\begin{array}{l}72.9 \\
(2.3) \\
79.4 \\
(2.0)\end{array}$ & $\begin{array}{l}12.7 \\
(2.1) \\
14.6 \\
(1.2)\end{array}$ & $\begin{array}{l}\text { EG, } 6 \text { months, } 3 \text { times a week, } \\
1 \text { h a day to stimulate aerobic } \\
\text { endurance, strength, balance, } \\
\text { motor coordination, agility and } \\
\text { flexibility; } 4,680 \text { min in total; } \\
\text { CG, care as usual }\end{array}$ & $\begin{array}{l}\text { Timed up and go } \\
\text { Berg balance scale }\end{array}$ & $\begin{array}{l}<0.05 \\
<0.05\end{array}$ & $\begin{array}{l}0.89 \\
3.02\end{array}$ & n.a. & $2 \mathrm{~B}$ & $\begin{array}{l}\text { Report. } 6 \\
\text { Ext. val. } 1 \\
\text { Int. val. bias } 4 \\
\text { Int. val. conf. } 0 \\
\text { Total } 11\end{array}$ \\
\hline $\begin{array}{l}\text { Hageman } \\
\text { and } \\
\text { Thomas } \\
\text { [29], 2002 }\end{array}$ & $\begin{array}{l}\text { Case } \\
\text { series }\end{array}$ & $\begin{array}{l}26 \\
88 \%\end{array}$ & $\begin{array}{l}79.2 \\
(6.6)\end{array}$ & $\begin{array}{l}18.0 \\
(6.2)\end{array}$ & $\begin{array}{l}6 \text { weeks, } 2 / 3 \text { times a week; resis- } \\
\text { tance training using thera bands; } \\
12 \text { different exercises aimed at } \\
\text { improving lower-limb strength, } \\
1 \text { set of } 15 \text { repetitions; } \\
2,700 \text { repetitions in total; } \\
\text { moderate intensity }\end{array}$ & $\begin{array}{l}\text { Gait speed free } \\
\text { Gait speed fast } \\
\text { Timed up and go }\end{array}$ & $\begin{array}{l}0.128 \\
0.045 \\
0.753\end{array}$ & $\begin{array}{l}0.20 \\
0.19 \\
0.06\end{array}$ & n.a. & 4 & $\begin{array}{l}\text { Report. } 6 \\
\text { Ext. val. } 1 \\
\text { Int. val. bias } 2 \\
\text { Int. val. conf. } 0 \\
\text { Total } 9\end{array}$ \\
\hline $\begin{array}{l}\text { Kwak } \\
\text { et al. } \\
\text { [37], } 2008\end{array}$ & $\mathrm{RCT}$ & $\begin{array}{l}\text { EG } 15 \\
100 \% \\
\text { CG } 15 \\
100 \%\end{array}$ & $\begin{array}{l}79.7 \\
(6.6) \\
82.3 \\
(7.1)\end{array}$ & $\begin{array}{l}14.5 \\
(5.3) \\
13.5 \\
(7.0)\end{array}$ & $\begin{array}{l}\mathrm{EG}, 12 \text { months, } 2 / 3 \text { times } \\
\text { a week, } 30-40 \mathrm{~min} \text {; start at } 30 \% \\
\mathrm{VO}_{2 \text { max. }} \text { up to } 60 \% \mathrm{VO}_{2} \text { max. thera } \\
\text { bands, Swiss ball, shoulder wheel, } \\
\text { staircase; } 4,550 \text { min in total; } \\
\text { CG, care as usual }\end{array}$ & $\begin{array}{l}\text { 6-min walk test } \\
\text { Muscle strength } \\
\text { Muscle endurance } \\
\text { Balance } \\
\text { ADL }\end{array}$ & $\begin{array}{l}<0.01 \\
<0.01 \\
<0.01 \\
<0.05 \\
<0.01\end{array}$ & $\begin{array}{l}1.75 \\
1.20 \\
1.03 \\
0.25 \\
1.32\end{array}$ & n.a. & $2 \mathrm{~B}$ & $\begin{array}{l}\text { Report. } 5 \\
\text { Ext. val. } 3 \\
\text { Int. val. bias } 4 \\
\text { Int. val. conf. } 2 \\
\text { Total } 14\end{array}$ \\
\hline $\begin{array}{l}\text { Kuiack } \\
\text { et al. } \\
{[25], 2004}\end{array}$ & $\begin{array}{l}\text { Case } \\
\text { series }\end{array}$ & $\begin{array}{l}8 \\
63 \%\end{array}$ & $\begin{array}{l}79 \\
63-88^{c}\end{array}$ & $\begin{array}{l}17 \\
13-20^{c}\end{array}$ & $\begin{array}{l}12 \text { weeks, } 2 \text { days a week; } \\
\text { resistance training } 3 \text { sets of } \\
8 \text { repetitions for } 5 \text { exercises; } \\
\text { aimed at improving leg extension, } \\
\text { shoulder press, hip abductor/ad- } \\
\text { ductor, chest/back and abdomen; } \\
2,880 \text { repetitions in total }\end{array}$ & $\begin{array}{l}\text { Gait speed } \\
\text { Leg extension } \\
\text { Hip abductor } \\
\text { Stair climb } \\
\text { Chair rise } \\
\text { Balance parallel } \\
\text { Balance semitandem } \\
\text { Balance tandem }\end{array}$ & $\begin{array}{l}\text { n.s. } \\
<0.01 \\
<0.01 \\
\text { n.s. } \\
\text { n.s. } \\
\text { n.s. } \\
\text { n.s. } \\
\text { n.s. }\end{array}$ & $\begin{array}{r}0.18 \\
1.95 \\
3.33 \\
1.18 \\
0.22 \\
1.83 \\
2.00 \\
-0.24\end{array}$ & $\begin{array}{l}\text { Per- } \\
\text { protocol }\end{array}$ & 4 & $\begin{array}{l}\text { Report. } 4 \\
\text { Ext. val. } 1 \\
\text { Int. val. bias } 4 \\
\text { Int. val. conf. } 1 \\
\text { Total } 10\end{array}$ \\
\hline $\begin{array}{l}\text { Netz } \\
\text { et al. } \\
{[30], 2007}\end{array}$ & RCT & $\begin{array}{l}\text { EG } 15 \\
\text { CG } 14\end{array}$ & $\begin{array}{l}76.9 \\
(6.7)\end{array}$ & $\begin{array}{l}13.3 \\
(5.8)\end{array}$ & $\begin{array}{l}\text { EG, } 12 \text { weeks, twice a week, } \\
45 \text { min ( } 1,080 \text { min in total); } \\
\text { range of motion, strength, } \\
\text { coordination, balance exercises } \\
\text { for upper and lower body, } \\
\text { group of } 13-15 \text { participants; } \\
\text { CG, care as usual }\end{array}$ & $\begin{array}{l}\text { Timed up and go } \\
\text { Sit-to-stand test }\end{array}$ & $\begin{array}{l}\text { n.s. } \\
\text { n.s. }\end{array}$ & $\begin{array}{l}-0.25 \\
-0.02\end{array}$ & $\begin{array}{l}\text { Per- } \\
\text { protocol }\end{array}$ & $2 \mathrm{~B}$ & $\begin{array}{l}\text { Report. } 7 \\
\text { Ext. val. } 1 \\
\text { Int. val. bias } 5 \\
\text { Int. val. conf. } 4 \\
\text { Total } 17\end{array}$ \\
\hline $\begin{array}{l}\text { Pomeroy } \\
\text { et al. } \\
{[26], 1999}\end{array}$ & RCT & $\begin{array}{l}\text { EG } 43 \\
74 \% \\
\text { CG } 38 \\
74 \%\end{array}$ & $\begin{array}{l}82.0 \\
(8.0) \\
81.8 \\
(8.4)\end{array}$ & n.a. & $\begin{array}{l}\text { EG, } 10 \text { half-hour sessions } \\
\text { of exercise to improve balance, } \\
\text { strength, range of motion; } \\
300 \text { min in total; } \\
\text { CG, nonphysical activities }\end{array}$ & $\begin{array}{l}\text { SMA } \\
\text { 2-min walk test }\end{array}$ & $\begin{array}{l}0.017 \\
0.048\end{array}$ & $\begin{array}{l}\text { n.a. } \\
\text { n.a. }\end{array}$ & $\begin{array}{l}\text { Intention- } \\
\text { to-treat }\end{array}$ & 2B & $\begin{array}{l}\text { Report. } 5 \\
\text { Ext. val. } 1 \\
\text { Int. val. bias } 5 \\
\text { Int. val. conf. } 2 \\
\text { Total } 13\end{array}$ \\
\hline
\end{tabular}


Table 1 (continued)

\begin{tabular}{|c|c|c|c|c|c|c|c|c|c|c|c|}
\hline Author & Design & $\begin{array}{l}\mathrm{n} \text { and } \\
\% \text { ? }\end{array}$ & $\begin{array}{l}\text { Age } \\
\text { years }^{\mathrm{a}}\end{array}$ & MMSE $^{\mathrm{a}}$ & Intervention & $\begin{array}{l}\text { Functional } \\
\text { outcome } \\
\text { measure }\end{array}$ & $\begin{array}{l}\mathrm{p} \\
\text { value }\end{array}$ & $\begin{array}{l}\text { Cohens' } \\
\text { d }\end{array}$ & Analysis & $\begin{array}{l}\text { Sackett } \\
\text { level of } \\
\text { evidence }\end{array}$ & $\begin{array}{l}\text { DB } \\
\text { checklist }\end{array}$ \\
\hline $\begin{array}{l}\text { Rolland } \\
\text { et al. } \\
\text { [31], } 2007\end{array}$ & RCT & $\begin{array}{l}\text { EG } 67 \\
72 \% \\
\text { CG } 67 \\
79 \%\end{array}$ & $\begin{array}{l}82.8 \\
(7.8) \\
83.1 \\
(7.0)\end{array}$ & $\begin{array}{l}9.7 \\
(6.8) \\
7.0 \\
(6.4)\end{array}$ & $\begin{array}{l}\text { EG, } 12 \text { months, twice a week, } \\
60 \text { min, } 88 \text { sessions in total; } \\
\text { aerobic strength, flexibility and } \\
\text { balance training; encouraged } \\
\text { to walk fast; strength training } \\
\text { focused on the lower extremity; } \\
5,280 \text { min in total; } \\
\text { CG, care as usual }\end{array}$ & $\begin{array}{l}\text { Gait speed } \\
\text { Up and go } \\
\text { 1-leg balance } \\
\text { ADL }\end{array}$ & $\begin{array}{l}0.002 \\
0.31 \\
0.34 \\
0.02\end{array}$ & $\begin{array}{c}0.32 \\
-0.10 \\
\text { n.a. } \\
0.22\end{array}$ & $\begin{array}{l}\text { Intention } \\
\text { to treat }\end{array}$ & $2 \mathrm{~B}$ & $\begin{array}{l}\text { Report. } 11 \\
\text { Ext. val. } 1 \\
\text { Int. val. bias } 6 \\
\text { Int. val. conf. } 5 \\
\text { Total } 23\end{array}$ \\
\hline $\begin{array}{l}\text { Santana- } \\
\text { Sosa et al. } \\
{[34], 2008}\end{array}$ & RCT & $\begin{array}{l}\text { EG } 8 \\
63 \% \\
\text { CG } 8 \\
63 \%\end{array}$ & $\begin{array}{l}76 \\
(4) \\
73 \\
(4)\end{array}$ & $\begin{array}{l}20.1 \\
(2.3) \\
19.9 \\
(1.7)\end{array}$ & $\begin{array}{l}\text { EG, } 12 \text { weeks, } 3 \text { times a week } \\
75 \text { min; joint mobility, resistance } \\
\text { and coordination exercises; } \\
\text { individualized; } 2,700 \text { min in total; } \\
\text { CG, care as usual }\end{array}$ & $\begin{array}{l}\text { Timed up and go } \\
\text { 2-min step test } \\
\text { Tinetti } \\
\text { Sit-to-stand test } \\
\text { ADL Katz } \\
\text { ADL Barthel }\end{array}$ & $\begin{array}{r}<0.001 \\
0.002 \\
<0.001 \\
<0.001 \\
0.019 \\
<0.001\end{array}$ & $\begin{array}{l}2.37 \\
0.58 \\
3.59 \\
3.14 \\
\text { n.a. } \\
\text { 5.06 }\end{array}$ & n.a. & $2 \mathrm{~B}$ & $\begin{array}{l}\text { Report. } 6 \\
\text { Ext. val. } 1 \\
\text { Int. val. bias } 5 \\
\text { Int. val. conf. } 5 \\
\text { Total } 17\end{array}$ \\
\hline $\begin{array}{l}\text { Schnelle } \\
\text { et al. } \\
{[27], 1995}\end{array}$ & RCT & $\begin{array}{l}\text { EG } 36 \\
\text { CG } 40\end{array}$ & $\begin{array}{l}85.1 \\
(8.2)\end{array}$ & 11.6 & $\begin{array}{l}\text { EG, } 8 \text { weeks, } 5 \text { times a week, } \\
4 \text { sessions a day; repetition of ex- } \\
\text { ercises specific to functional skills, } \\
\text { i.e. sit-to-stand exercises, walking } \\
\text { a little bit further when going to } \\
\text { the toilet; CG, care as usual }\end{array}$ & $\begin{array}{l}\text { Gait speed } \\
\text { Walking endurance }\end{array}$ & $\begin{array}{r}0.06 \\
<0.05\end{array}$ & $\begin{array}{l}\text { n.a. } \\
0.50\end{array}$ & n.a. & $2 \mathrm{~B}$ & $\begin{array}{l}\text { Report. } 4 \\
\text { Ext. val. } 2 \\
\text { Int. val. bias } 3 \\
\text { Int. val. conf. } 3 \\
\text { Total } 12\end{array}$ \\
\hline $\begin{array}{l}\text { Steinberg } \\
\text { et al. } \\
{[28], 2009}\end{array}$ & RCT & $\begin{array}{l}\text { EG } 14 \\
71.4 \% \\
\text { CG } 13 \\
69.2 \%\end{array}$ & $\begin{array}{l}76.5 \\
(3.9) \\
74.0 \\
(8.1)\end{array}$ & $\begin{array}{l}20.1 \\
(5.1) \\
15.5 \\
(5.4)\end{array}$ & $\begin{array}{l}\text { EG, } 12 \text { weeks, get } 6 \text { aerobic points, } \\
4 \text { strength points and } 4 \text { balance } \\
\text { points a week; with different } \\
\text { exercises, aiming to improve } \\
\text { aerobic strength and balance; } \\
\text { CG, home safety review }\end{array}$ & $\begin{array}{l}\text { Gait speed } \\
\text { YPAS } \\
\text { Sit-to-stand test }\end{array}$ & $\begin{array}{l}0.77 \\
0.76 \\
0.22\end{array}$ & $\begin{array}{l}\text { n.a. } \\
\text { n.a. } \\
\text { n.a. }\end{array}$ & $\begin{array}{l}\text { Intention- } \\
\text { to-treat }\end{array}$ & $2 \mathrm{~B}$ & $\begin{array}{l}\text { Report. } 6 \\
\text { Ext. val. } 0 \\
\text { Int. val. bias } 4 \\
\text { Int. val. conf. } 4 \\
\text { Total } 14\end{array}$ \\
\hline $\begin{array}{l}\text { Tappen } \\
\text { et al. } \\
\text { [32], } 2000\end{array}$ & RCT & $\begin{array}{l}\text { EG } 20 \\
\text { CG } 22\end{array}$ & $\begin{array}{l}84.3 \\
(7.5) \\
89.6 \\
(6.5)\end{array}$ & $\begin{array}{l}10.8 \\
(6.0) \\
12.5 \\
(5.9)\end{array}$ & $\begin{array}{l}\text { EG, } 16 \text { weeks, } 3 \text { times a week, } \\
30 \text { min; walking and conversation; } \\
1,440 \text { min of walking in total; } \\
\text { CG, conversation only }\end{array}$ & 6-min walk & 0.001 & 0.31 & $\begin{array}{l}\text { Per- } \\
\text { protocol }\end{array}$ & $2 \mathrm{~B}$ & $\begin{array}{l}\text { Report. } 7 \\
\text { Ext. val. } 1 \\
\text { Int. val. bias } 5 \\
\text { Int. val. conf. } 3 \\
\text { Total } 16\end{array}$ \\
\hline $\begin{array}{l}\text { Thomas } \\
\text { and } \\
\text { Hageman } \\
{[36], 2003}\end{array}$ & $\begin{array}{l}\text { Case } \\
\text { series }\end{array}$ & 28 & $\begin{array}{l}80.0 \\
(5.6)\end{array}$ & $\begin{array}{l}17.8 \\
(7.2)\end{array}$ & $\begin{array}{l}6 \text { weeks, } 3 \text { sessions a week; resis- } \\
\text { tance exercise using thera bands; } \\
1 \text { set of } 15 \text { repetitions for } 12 \text { exer- } \\
\text { cises aimed at improving lower- } \\
\text { limb strength; } 2,700 \text { repetitions } \\
\text { in total; moderate intensity }\end{array}$ & $\begin{array}{l}\text { Gait speed free } \\
\text { Gait speed fast } \\
\text { Timed up and go } \\
\text { Knee extensor right } \\
\text { Knee extensor left } \\
\text { Sit-to-stand test }\end{array}$ & $\begin{array}{l}0.19 \\
0.06 \\
0.71 \\
0.44 \\
0.46 \\
0.02\end{array}$ & $\begin{array}{r}-0.11 \\
0.10 \\
0.85 \\
0.14 \\
-0.04 \\
0.50\end{array}$ & n.a. & 4 & $\begin{array}{l}\text { Report. } 7 \\
\text { Ext. val. } 2 \\
\text { Int. val. bias } 4 \\
\text { Int. val. conf. } 2 \\
\text { Total } 15\end{array}$ \\
\hline $\begin{array}{l}\text { Toulotte } \\
\text { et al. } \\
\text { [35], } 2003\end{array}$ & $\mathrm{RCT}$ & $\begin{array}{l}\text { EG } 10 \\
\text { CG } 10\end{array}$ & $\begin{array}{l}81.0 \\
(5.6) \\
81.9 \\
(4.1)\end{array}$ & $\begin{array}{l}14.7 \\
(7.6) \\
18.0 \\
(5.4)\end{array}$ & $\begin{array}{l}\text { EG, } 16 \text { weeks, } 2 \text { sessions a week, } \\
45 \text { min; muscular strength, static } \\
\text { and dynamic, balance, flexibility } \\
\text { and proprioception, exercises; } \\
5 \text { persons a group; } 1,440 \text { min in } \\
\text { total; CG, care as usual }\end{array}$ & $\begin{array}{l}\text { Gait speed } \\
\text { Timed up and go } \\
\text { Balance }\end{array}$ & $\begin{array}{r}0.015 \\
0.001 \\
<0.01\end{array}$ & $\begin{array}{l}\text { n.a. } \\
\text { n.a. } \\
\text { n.a. }\end{array}$ & n.a. & $2 \mathrm{~B}$ & $\begin{array}{l}\text { Report. } 8 \\
\text { Ext. val. } 0 \\
\text { Int. val. bias } 4 \\
\text { Int. val. conf. } 3 \\
\text { Total } 15\end{array}$ \\
\hline
\end{tabular}

MMSE = Mini Mental State Examination; report. = reporting; ext. val. = external validity; int. val. bias = internal validity bias; int. val. conf. = internal validity confounding; $\mathrm{EG}$ = exercise group; $\mathrm{CG}=$ control group; n.a. = not available; n.s. = not significant; SMA = Southampton Mobility Assessment; $\mathrm{VO}_{2 \text { max. }}=$ maximum oxygen volume; YPAS = Yale Physical Activity Survey. Figures in parentheses indicate standard deviations.

${ }^{a}$ Mean and standard deviations unless otherwise noted.

${ }^{b}$ Score on the Saint Louis University Mental Status Examination. ${ }^{c}$ Range. 
Table 2. Rate of enrollment, number of dropouts and participation rate

\begin{tabular}{|c|c|c|c|c|c|}
\hline Study & Eligible & $\begin{array}{l}\text { Rate of } \\
\text { enrollment }\end{array}$ & $\mathrm{n}_{\text {pre }}$ & Dropouts & Participation rate \\
\hline Aman and Thomas [22], 2009 & 55 & $91 \%$ & 50 & 10; did not want to exercise & $30.7 \%$ \\
\hline Arkin [33], 2003 & n.a. & n.a. & 24 & n.a. & $\begin{array}{l}\text { student } 100 \% \\
\text { caregivers } 60-80 \%\end{array}$ \\
\hline Binder [23], 1995 & 34 & $100 \%$ & 34 & $\begin{array}{l}9 ; 8 \text { did not want to exercise, } \\
1 \text { responsible proxy revoked consent }\end{array}$ & $75 \%$ \\
\hline Christafoletti et al. [24], 2008 & 63 & $86 \%$ & 54 & $\begin{array}{l}13 ; 3 \text { died, } 3 \text { for unknown reasons, } \\
3 \text { medication use, } 4 \text { clinical instability }\end{array}$ & n.a. \\
\hline Hageman and Thomas [29], 2002 & 75 & $35 \%$ & 26 & n.a. & n.a. \\
\hline Kwak et al. [37], 2008 & n.a. & n.a. & 30 & n.a. & n.a. \\
\hline Kuiack et al. [25], 2004 & 13 & $85 \%$ & 11 & 3; unrelated to training program & $100 \%$ \\
\hline Netz et al. [30], 2007 & 50 & $58 \%$ & 29 & 4; being moved, getting help at home & $>70 \%$ \\
\hline Pomeroy et al. [26], 1999 & 91 & $89 \%$ & 81 & n.a. & $80 \%$ \\
\hline Rolland et al. [31], 2007 & 242 & $55 \%$ & 134 & $\begin{array}{l}24 \text {; dead } 15 \text {, change of institution } 8 \text {, } \\
\text { discontinued } 4\end{array}$ & $\begin{array}{l}19.4 \%>2 / 3 \text { of sessions } \\
28.4 \% 1 / 3-2 / 3 \text { of sessions } \\
41.8 \%<1 / 3 \text { of sessions } \\
10.4 \% 0 \text { sessions }\end{array}$ \\
\hline Santana-Sosa et al. [34], 2008 & n.a. & n.a. & 16 & n.a. & $98.9 \%$ \\
\hline Schnelle et al. [27], 1995 & 99 & $95 \%$ & 94 & 18; death, transfer, hospitalization & $75 \%$ \\
\hline Steinberg et al. [28], 2009 & 30 & $90 \%$ & 27 & n.a. & overall $75 \%$ \\
\hline Tappen et al. [32], 2000 & n.a. & n.a. & 71 & 6 & walking + conversation $75 \%$ \\
\hline Thomas and Hageman [36], 2003 & n.a. & n.a. & n.a. & n.a. & n.a. \\
\hline Toulotte et al. [35], 2003 & n.a. & n.a. & 20 & n.a. & n.a. \\
\hline
\end{tabular}

$\mathrm{n}_{\text {pre }}=$ Number of participants at baseline; n.a. = not available.

mum methodological score: reporting, $60.2 \%$; external validity, $36.5 \%$; internal validity bias, $66.1 \%$; internal validity confounding, $51.6 \%$. There were 6 nonrandomized trials of level 4 evidence and an average score of 11 points on the DB (range 4-19). These 6 studies scored $40.7 \%$ of the maximum methodological score: reporting $50 \%$, external validity $44.3 \%$, internal validity bias $44.3 \%$, internal validity confounding $19.5 \%$.

Five studies performed a per-protocol analysis, 3 performed an intention-to-treat analysis, and 1 study performed both analyses; 7 studies did not present this information.

\section{Participant Population}

Ten studies provided information about eligible candidates; 7 studies showed a rate of enrollment of $>85 \%$ [22-28], 3 studies had a rate between 35 and 58\% [29-31]. Eight studies provided the number of dropouts, of which 6 studies had a dropout rate between 18 and 27\% [22-25, $27,31] ; 1$ had a dropout rate of $14 \%$ [30] and the last one of $8 \%$ [32]. The reasons for dropouts were hospitalization, death, being moved and refusing to exercise. Not one single study reported dropouts due to negative adverse effects of the intervention. No relationship could be found between the duration of the intervention, i.e. the number of weeks or months, and the dropout rate $(\mathrm{r}=$ $0.301, p=0.468$, Spearman's $\rho$ ) $[22-25,27,30-32]$. However, there was a moderate to strong, positive and significant correlation $(r=0.829 ; \mathrm{p}=0.042$, Spearman's $\rho$ ) between the level of global cognitive functioning, measured with the Mini Mental State Examination (MMSE), and the dropout rate [23-25, 30-32]. In other words, if participants had a higher score on the MMSE, the chance that they dropped out of the study increased. The participation rate, calculated over 8 studies $[23,25,27,28$, $30,32-34]$, was $81.4 \%$. In contrast to the dropout rate, the participation rate is higher if the cognitive level increased (7 studies, $r=0.729, p=0.063$, Spearman's $\rho$ ) $[23,25,27$, $28,31,32,34]$. One study found that the participation rate increased if students were responsible for the execution of the intervention instead of the family caregivers [32]. A second study showed that a conversation during a walking intervention has a beneficial influence on the participation rate [32]. However, 1 study contrasted 
sharply with the other studies [31]. In the latter study, only $19.4 \%$ of the participants performed more than two thirds of the sessions, and around $50 \%$ of the participants performed more than one third of the sessions. However, $10 \%$ did not participate in any exercise. The participants in this latter study had the lowest score on cognition, and the inclusion and exclusion criteria were not very selective [31].

\section{Effects of Exercise}

Effect of Physical Interventions on Gait Speed, Endurance, Functional Mobility, Lower-Extremity Strength and Balance

Gait speed was assessed in 6 different studies, 2 level $2 \mathrm{~B}$ studies [31, 35], with, respectively, 23 and 5 on the DB questionnaire, and 4 level 4 studies, with respectively 19 , 9,10 and 15 on the DB $[22,25,29,36]$. Only in 1 level $2 B$ study could the ES be calculated $(\mathrm{d}=0.32, \mathrm{p}=0.002)[31$; in the other level $2 \mathrm{~B}$ study, a significant improvement in gait speed $(\mathrm{p}=0.015)$ was observed [35]. A moderate ES $(\mathrm{d}=0.50, \mathrm{p}<0.001)$ was obtained in a level 4 study [22]. The latter 3 studies used multicomponent interventions (i.e. a combination of strength, endurance and flexibility training) with durations ranging from 3 weeks to 12 months. In contrast, level 4 studies with progressive resistance training of 6 and 12 weeks found almost no effect on normal gait speed $(\mathrm{d} \leq 0.2)[25,29,36]$. Concerning fast gait speed, a low ES was found after progressive resistance training $(\mathrm{d}=0.19, \mathrm{p}=0.045)$ [29]. No information was available on the effect of multicomponent interventions on fast gait speed.

Endurance was assessed in 5 studies, 4 level 2B studies, which had between 13 and 17 points on the DB [26, 32, 34,37 , and 1 level 4 study with 4 points on the DB [33]. All studies found a positive effect of physical activity on the endurance capacity $[26,32-34,37]$. In the multicomponent interventions the ES was larger, if the duration of the intervention was longer, ranging from $\mathrm{d}=3.79$ after an intervention of $1-4$ years, to $d=0.58$ in an intervention of 12 weeks $[33,34,37]$. A solely walking intervention of 16 weeks had the lowest ES $(\mathrm{d}=0.31)$ [32]. No information is available on the effect of progressive resistance training on endurance.

Functional mobility was assessed in 8 studies, 6 level 2B studies, which were all based on multicomponent interventions [24, 26, 30, 31, 34, 35], and 2 level 4 progressive resistance training interventions $[29,36]$. Only from 2 level 2B studies could ES be calculated and appeared to be large $(\mathrm{d}=0.89, \mathrm{p}<0.05 ; \mathrm{d}=2.37, \mathrm{p}<0.001)[24,34]$. From the remaining 4 studies, 2 did find a significant im- provement [26, 35], and 2 studies did not [30,31]. Possible explanations for the negative results are the short duration of the intervention [30] or the qualitative score which might be less suitable than quantitative scores in this specific population [31]. However, it should be noted that the studies without a significant improvement had a higher score on the $\mathrm{DB}$, which indicates that their methodological quality is higher (17 and 23, compared to 17, 15, 13 and 11 points, respectively). Both level 4 studies did not find an improvement on functional mobility. These 2 studies used progressive resistance training and had a duration of 6 weeks $[29,36]$.

Lower-extremity strength was assessed in 7 different studies. There were 3 level $2 \mathrm{~B}$ studies with a range of 1317 points on the DB which used multicomponent interventions [30,34,37], and 4 level 4 studies with a range of $4-15$ points on the DB $[23,25,33,36]$. Two level $2 B$ studies found large ESs on lower-extremity strength $(\mathrm{d}=3.14$; $\mathrm{d}=1.20)[34,37]$. The other level $2 \mathrm{~B}$ study did not find any effect $(\mathrm{d}=-0.02)$ [30]. This latter study had the fewest minutes spent on the intervention from all level 2B studies. There were 2 level 4 studies which used a multicomponent intervention [23,33] and 2 that only used progressive resistance training $[25,36]$. Although the interventions differed, the largest ESs were found in the interventions with the longest duration. A multicomponent intervention of $1-4$ years $(\mathrm{d}=2.73)$ [33] outperformed a multicomponent intervention of 8 weeks $(\mathrm{d}=0.34)$ [23]. In accordance, a progressive resistance training of 12 weeks $(\mathrm{d}=1.95)$ [25] outperformed a progressive resistance training of 6 weeks $(\mathrm{d}=0.14)$ [36].

Balance was assessed in 7 studies, 5 multicomponent level 2B studies [24, 31, 34, 35, 37] and 2 level 4 studies with one using multicomponent intervention and the other using a progressive resistance training [23, 25]. Four of the 5 level 2B studies found a positive effect on balance with ES varying from low to very high $(\mathrm{d}=0.247-$ 3.59). The studies with a longer duration or a higher frequency had a higher ES. In 1 level 2B study, probably caused by the use of a qualitative score instead of a quantitative one, no significant improvement was found [31]. From the 2 level 4 studies, 1 multicomponent study found a moderate ES after a multicomponent intervention of 8 weeks [23]. The other study used progressive resistance training and found a high but nonsignificant ES ( $\mathrm{d}=$ 2.00) after 12 weeks [25].

The Effect of Physical Interventions on BADL

In the majority of the studies selected for this review, BADL was not assessed. Only 4 studies investigated the 
Table 3. Mean ESs and mean methodological quality weighted with sample size

\begin{tabular}{llll}
\hline Outcome measures & $\begin{array}{l}\text { Number } \\
\text { of studies }\end{array}$ & ES & $\begin{array}{l}\text { Methodological } \\
\text { quality }\end{array}$ \\
\hline Gait speed (normal) & 6 & $0.29(-0.11$ to 0.50$)$ & $18.8(9-23)$ \\
Gait speed (fast) & 2 & $0.14(0.10$ to 0.19$)$ & $12.1(9-15)$ \\
Endurance & 5 & $1.08(0.31$ to 3.79$)$ & $12.7(4-17)$ \\
Functional mobility & 6 & $0.28(-0.25$ to 2.37$)$ & $18.2(9-23)$ \\
Lower-extremity strength & 7 & $0.85(-0.04$ to 3.14$)$ & $13.3(4-17)$ \\
Balance & 5 & $1.76(-0.24$ to 3.59$)$ & $12.1(9-17)$ \\
ADL & 4 & $0.68(0.11$ to 5.06$)$ & $20.5(14-23)$ \\
\hline
\end{tabular}

ES = Effect size (Cohen's d). Methodological quality score based on the DB questionnaire, theoretical range 0 (lowest quality) to 26 (highest quality). Figures in parentheses indicate ranges. effects of exercise on BADL; all these studies used multicomponent interventions. There were 3 level $2 \mathrm{~B}$ studies, 2 studies found a very high ES $(\mathrm{d}=1.32 ; \mathrm{d}=5.78)[34,37$, the other had a low ES $(\mathrm{d}=0.22)$ [31]. The latter study achieved the highest points on the $\mathrm{DB}, 23$ compared to 14 and 17 for the first 2 studies. The positive effects of physical activity on BADL were observed in participants with mild (mean MMSE 20), moderate (mean MMSE 14) and severe dementia (mean MMSE 8.8). The interventions ranged from 12 weeks to 12 months. One study, level 4, did not find a significant improvement in BADL performance. However, this latter study had a duration of 3 weeks with $270 \mathrm{~min}$ of exercise in total [22].

The improvements in BADL were supported by improvements in physical functioning in 2 studies [34, 37]. The third study did find an improvement in gait speed, but not in balance and mobility. However, the latter finding might be caused by the use of qualitative instead of quantitative scores [31].

\section{The Overall Effect of Physical Activity}

To compare overall effects, the mean ESs of the different outcome measures were calculated (table 3). It is interesting that the extreme ESs, very high or low, correspond with a relatively low methodological quality. Overall, the moderate ESs are found in studies with more acceptable methodological scores.

\section{Discussion}

The first goal of this systematic review was to investigate whether physical activity improves physical functioning, i.e. gait speed, functional mobility, balance, endurance, lower-extremity strength and BADL in elderly subjects with dementia. A second goal was to formulate guidelines for the most effective training program.

Before we are able to answer these questions properly, it is important to get more insight into the participants. Are these persons representative of the total population of elderly persons with dementia? Therefore, it is necessary to know how many people were interested in participating, and how many were allowed to enroll in the intervention program. From this latter group, it is well worth knowing how they complied with the protocol. We found that the majority of the participants were willing to participate. Seven out of 10 studies found a rate of enrollment of at least $85 \%$. Three studies had a lower rate of inclusion probably caused by more specific inclusion and exclusion criteria regarding cognitive functioning or mobility. Of the participants who started with the intervention, about 20-25\% dropped out for different reasons (e.g. death, hospitalization, refusal). Not one dropped out due to adverse effects of the intervention. It is remarkable that participants with higher scores on the MMSE have higher dropout rates, but also higher participation rates. We speculate that people with higher scores on the MMSE have better memories and stronger wills of their own. Therefore, a part of this group will indicate that they do not want to continue the program (dropout), but another part will decide, by themselves, that they do want to continue, which results in higher participation. From the participants in the interventions the average participation rate was around $80 \%$, though large differences were found. The participation rate could be increased by talking during the interventions or by using students to guide the participants, instead of family caregivers [32,33]. The results regarding rates of enrollment, dropouts and participation rates do differ between the different studies, but not enough information is provided to analyze this 
difference in detail. In the majority of studies, physical exercise is an activity which was performed and sustained by most elderly individuals. Generally, the rates of enrollment and the participation rates suggest that elderly subjects with dementia like to take part in interventions involving physical activity. Although this is important information, less than $70 \%$ of the included studies provided information regarding rate of enrollment, dropout rates and participation rates. We recommend that future studies provide this information.

Elderly subjects with dementia can take part in physical activity interventions, but they also benefit from ordinary exercise. Physical activity interventions in elderly with dementia lead to an improvement in physical performance, as presented by overall ESs (table 3). The largest improvements on gait speed, functional mobility and balance were seen after multicomponent interventions, and not after progressive resistance training. A positive improvement was also found in endurance after multicomponent interventions. Lower-extremity strength increased after these, as well as progressive resistance training, but it is unclear which interventions lead to the best outcome. In most cases, the largest improvements in physical functioning were found after interventions with the largest training volume. However, it should be noted that only 16 studies are incorporated in this systematic review, each with different durations, ranging from 3 weeks to 4 years, and different components within their interventions. Therefore, it is important to get more insight into the effects of different durations, different training volume, and the different components within each intervention (e.g. exercises, intensity), in order to understand the mechanism behind the intervention. With the presented data we are unable to assess the quality of the different components of each intervention by themselves. Therefore, it is not possible to determine the dose-response relationship in detail, and consequently, we can only provide more general guidelines. Based on the presented data, the largest improvements are found after interventions with a duration of at least 12 weeks, with a frequency of 3 times a week, for 45-60 min per session. Improvements were seen amongst participants with mild [34], moderate [37] and severe [31] dementia. This indicates that physical activity is beneficial in each stage of dementia. Our findings are in line with physical activity intervention studies in elderly without cognitive decline, and elderly in long-term care institutions $[38,39]$.

The effect of physical activity on BADL can be hypothesized using the model of the International Classifications of Functioning, Disability and Health. An improve- ment in body functions, such as gait speed, functional mobility, endurance, lower extremity strength and balance, can lead to an improvement in activities, such as BADL, which might lead to an increase in participation. However, BADL was only assessed in 4 studies. From these 4 studies, 3 with the largest training volume and highest methodological quality found improvements in BADL. These improvements were found in patients with mild, moderate and severe dementia. Among the 3 studies, 2 found an improvement in all the other physical measurements, which is in accordance with the relationship between physical performance and BADL. However, 1 study did not find improvements on all measurements; more specifically it did not find improvements on the qualitative scoring measurement (up and go, singlestance). It might be that, due to the characteristics of the population, floor effects appeared. Due to the small number and the mixed results, it is not possible to determine if an improvement in physical performance would mediate the effect of exercise on BADL. It could also be mediated by cognition, for example. Future studies should focus on the effect of physical activity interventions on physical functioning and BADL, and take other possible mediating factors, like cognition, and moderating factors, like depression, apathy and agitation, into account. This is necessary to get more insight into the mechanism by which physical activity works.

Although we tried to ensure that all studies referring to elderly subjects with dementia and physical activity were included in this review, we cannot rule out the possibility that we have missed some. For example, in the literature we found some studies that were performed in nursing homes, and we found studies where participants had a score of 24 or lower on the MMSE. However, when we were not absolutely sure that the participants of these latter studies had dementia, these studies were excluded. Therefore, we recommend that future studies try to describe their population as specifically as possible. Preferably, their descriptions should be supported with additional information, such as the etiology of the disease, a measurement of cognition like the MMSE [10], global assessment of functioning such as the Clinical Dementia Rating or the Global Deterioration Scale [40]. Moreover, the studies that were included in this review were, in general, of mediocre quality according to the DB list. For all the level 2B studies compiled, an average of $56 \%$ of the maximum methodological score was obtained. Especially on reporting, external validity and internal validity (confounding), information was missing. This indicates the urgent need for high-quality intervention studies. Anoth- 
Table 4. Summary of recommendations

\begin{tabular}{|c|c|}
\hline $\begin{array}{l}\text { Classifica- } \\
\text { tion }\end{array}$ & Recommendations \\
\hline $\begin{array}{l}\text { Method- } \\
\text { ological } \\
\text { issues }\end{array}$ & $\begin{array}{l}\text { - Provide detailed information about the diagnosis, cognition, participation rate of the participants, } \\
\text { dropouts and rate of enrollment } \\
\text { - Choose measurements that are sensitive for change, valid and reliable for the targeted population } \\
\text { - Present the data in such way that more calculations, like ES, can be performed }\end{array}$ \\
\hline $\begin{array}{l}\text { Questions } \\
\text { for further } \\
\text { research }\end{array}$ & $\begin{array}{l}\text { - Regarding the paucity of studies assessing BADL after physical exercise in elderly subjects with } \\
\text { dementia: how can exercise optimally influence BADL? } \\
\text { Dose-response relationship: what are the optimal duration, frequency and intensity? } \\
\text { - Type of activities: are some components more important than others, and what is the best } \\
\text { - combination of components? } \\
\text { Working mechanisms: can mediating factors be identified (e.g. cognition)? } \\
\text { - Intervention related to cognitive level: should an intervention be changed if the cognitive level } \\
\text { - changes? }\end{array}$ \\
\hline $\begin{array}{l}\text { Practical } \\
\text { guidelines }\end{array}$ & $\begin{array}{l}\text { - Offer exercise in all stages of dementia } \\
\text { - Use multicomponent interventions } \\
\text { - A duration of } 12 \text { weeks or more } \\
\text { - Exercise at least } 3 \text { times a week } \\
\text { - Exercise } 45-60 \mathrm{~min} / \mathrm{session}\end{array}$ \\
\hline
\end{tabular}

er important aspect is the choice of measurements. Elderly subjects with dementia are a very specific population, and probably for that reason the psychometric qualities of multiple measurements have not been studied thoroughly. We recommend that measurements are chosen in such a way that ESs can be calculated and floor effects should not be possible. It is important that measurements are sensitive for change in such a vulnerable population.

\section{Conclusion}

Physical activity can be beneficial in all stages of dementia. However, to get more insight into the mechanism underlying the effects of physical activity, we need more high-quality studies. It is of the utmost importance that specific information about the intervention is well documented, such as the characteristics of the participants, the intensity, duration and the different components. This information is necessary in order to interpret the external validity, possible confounders and the dose-response relationship in detail. Future studies should focus on the effect of physical activity interventions on physical functioning and on BADL, and should take other mediators, like cognition, into account. Based on the presented data, it can be concluded that multicomponent interven- tions with a high training volume lead to an improvement in physical performance and BADL, and outperform walking and progressive resistance training intervention in most aspects. The largest improvements were found after interventions with a duration of 12 weeks or more, and a frequency of 3 times a week, with 45-60 min a session. Practical guidelines and recommendations for future studies are summarized in table 4 .

\section{Acknowledgment}

The research reported in this paper was supported by a grant from the Open Ankh (GD 55.06 to C.G.B.).

\section{Disclosure Statement}

All authors declare that there are no conflicts of interest. 


\section{References}

$\checkmark 1$ Ferri CP, Prince M, Brayne C, Brodaty H, Fratiglioni L, Ganguli M, Hall et al: Global prevalence of dementia: a Delphi consensus study. Lancet 2005;366:2112-2117.

$\checkmark 2$ Geldmacher DS, Frolich L, Doody RS, Erkinjuntti T, Vellas B, Jones RW, Banerjee S, Lin $\mathrm{P}$, Sano M: Realistic expectations for treatment success in Alzheimer's disease. J Nutr Health Aging 2006; 10:417-429.

$\checkmark 3$ Schölzer-Dorenbosch CJM: Medicamenteuze behandeling van de ziekte van $\mathrm{Alz}$ heimer: de klinische praktijk in Nederland. Tijdschr Gerontol Geriatrie 2005;36:122126.

4 Lövheim H, Sandman PO, Karlsson S, Gustafson Y: Behavioral and psychological symptoms of dementia in relation to level of cognitive impairment. Int Psychogeriatr 2008;20:777-789.

$\checkmark 5$ Feldman $\mathrm{HH}$, van Baelen B, Kavanagh SM, Torfs KEL: Cognition, function, and caregiving time patterns in patients with mildto-moderate Alzheimer disease: a 12 month analysis. Alzheimer Dis Assoc Disord 2005; 19:29-36.

-6 Gaugler JE, Duval S, Anderson KA, Kane RL: Predicting nursing home admission in the US: a meta-analysis. BMC Geriatr 2007;7:13.

$\checkmark 7$ Iavarone A, Milan G, Vargas G, Lamenza F, De Falco C, Gallotta G, Postiglione A: Role of functional performance in diagnosis of dementia in elderly people with low educational level living in Southern Italy. Aging Clin Exp Res 2007;19:104-109.

$>8$ Galasko D, Kershaw PR, Schneider L, Zhu Y, Tariot PN: Galantamine maintains ability to perform activities of daily living in patients with Alzheimer's disease. J Am Geriatr Soc 2004;52:1070-1076.

9 Boersma F, van den Brink W, Deeg DJ, Eefsting JA, van Tilburg W: Survival in a population-based cohort of dementia patients: predictors and causes of mortality. Int J Geriatr Psychiatry 1999;14:748-753.

-10 Folstein MF, Folstein SE, McHugh PR: Mini mental state: a practical method for grading the cognitive state of patients for the clinician. J Psychiatr Res 1975;12:189-198.

-11 Feldman HH, Schmitt FA, Olin JT: Activities of daily living in moderate-to-severe Alzheimer disease: an analysis of the treatment effects of memantine in patients receiving stable donepezil treatment. Alzheimer Dis Assoc Disord 2006;4:263-268.

12 World Health Organization: International classification of functioning, disabilities, and health: ICF. Geneva, World Health Organization, 2001.

-13 Binder EF, Schechtmann KB, Ehsani AA, Steger-May K, Brown M, Sinacore DR, Yarasheski KE, Holloszy JO: Effects of exercise training on frailty in community-dwelling older adults: results of a randomized, controlled trial. J Am Geriatr Soc 2002;50: 1921-1928.
14 Vaitkevicius PV, Ebersold C, Shah MS, Gill NS, Katz RL, Narrett MJ, Applebaum GE, Parrish SM, O’Connor FC, Fleg JL: Effects of aerobic exercise training in communitybased subjects aged 80 and older: a pilot study. J Am Geriatr Soc 2002;50:2009-2013.

15 Yokoya T, Demura S, Sato S: Three-year follow-up of the fall risk and physical function characteristics of the elderly participating in a community exercise class. J Physiol Anthropol 2009;28:55-62.

16 Downs SH, Black N: The feasibility of creating a checklist for the assessment of the methodological quality both of randomized and non-randomised studies of health care interventions. J Epidemiol Community Health 1998;52:377-384.

17 Sackett DL, Straus SE, Richardson WS: Evidence-Based Medicine: How to Practice and Teach EBM, ed 2. Edinburgh, Churchill Livingstone, 2002.

18 Cohen J: Statistical Power Analysis for the Behavioral Sciences, ed 2. Hillsdale, Erlbaum, 1988.

19 Robey RR: Reporting point and interval estimates of effect-size for planned contrasts: fixed within effects analyses of variance. J Fluency Disord 2004;29:307-341.

20 Thalheimer W, Cook S: How to calculate effect sizes from published research articles: a simplified methodology. 2002. http://worklearning.com/effect_sizes.htm (accessed October 20, 2009).

21 Cohen J: A power primer. Psychol Bull 1992; 112:155-159.

22 Aman E, Thomas DR: Supervised exercise to reduce agitation in severely cognitively impaired persons. J Am Med Dir Assoc 2009; 10:271-276.

23 Binder EF: Implementing a structured exercise program for frail nursing home residents with dementia: issues and challenges. J Aging Physical Activity 1995;3:383-395.

24 Christafoletti G, Oliani MM, Gobbi S, Stella F, Gobbi LTB, Canineu PR: A controlled clinical trial on the effects of motor intervention on balance and cognition in institutionalized elderly patients with dementia. Clin Rehabil 2008;22:618-626.

25 Kuiack SL, Campbell WW, Evans WJ: A structured resistive training program improves muscle strength and power in elderly persons with dementia. Activities Adaptation Aging 2004;28:35-47.

26 Pomeroy VM, Warren CM, Honeycombe C Briggs RSJ, Wilkinson DG, Pickering RM, Stiener A: Mobility and dementia: is physiotherapy treatment during respite care effective? Int J Geriatr Psychiatry 1999;14:389397.

27 Schnelle JF, MacRae PG, Ouslander JG, Simmons SF, Nitta M: Functional incidental training, mobility performance and incontinence care with nursing home residents. J Am Geriatr Soc 1995;43:1356-1362.
28 Steinberg M, Leoutsakos JMS, Podewils LJ, Luketsos CG: Evaluation of a home-based exercise program in the treatment of $\mathrm{Alz}$ heimer's disease: the Maximizing Independence in Dementia (MIND) study. Int J Geriatr Psychiatry 2009;24:680-685.

29 Hageman PA, Thomas VS: Gait performance in dementia: the effects of a 6-week resistance training program in an adult day-care setting. Int J Geriatr Psychiatry 2002;17:329334.

30 Netz Y, Axelrad S, Argov E: Group physical activity for demented older adults feasibility and effectiveness. Clin Rehabil 2007;21:977986.

31 Rolland Y, Pillard F, Klapouszczak A, Reynish E, Thomas D, Andrieu S, Riviere D, Vellas B: Exercise program for nursing home residents with Alzheimer's disease: a 1-year randomized, controlled trial. J Am Geriatr Soc 2007;55:158-165

32 Tappen RM, Roach KE, Applegate EB, Stowell P: Effect of a combined walking and conversation intervention on functional mobility of nursing home residents with Alzheimer disease. Alzheimer Dis Assoc Disord 2000;14:196-201.

33 Arkin SM: Student-led exercise sessions yield significant fitness gains for Alzheimer patients. Am J Alzheimers Dis Other Demen 2003;18:159-170.

34 Santana-Sosa A, Barriopedro MI, LopezMojares LM, Perez M, Lucia A: Exercise training is beneficial for Alzheimer's patients. Int J Sports Med. 2008;29:845-850.

35 Toulotte C, Fabre C, Dangremont B, Lensel $G$, Thevenon A: Effects of physical training on the physical capacity of frail, demented patients with a history of falling: a randomized controlled trial. Age Ageing 2003;32: 67-73.

36 Thomas VS, Hageman PA: Can neuromuscular strength and functioning people with dementia be rehabilitated using resistanceexercise training? Results from a preliminary intervention study. J Gerontol Med Sci 2003;58A:746-751.

37 Kwak YS, Um SY, Son TG, Kim DJ: Effect of regular exercise on senile dementia patients. Int J Sports Med 2008;29:471-474.

38 Kolbe-Alexander TL, Lambert EV, Charlton KE: Effectiveness of a community based low intensity exercise program for older adults. J Nutr Health Aging 2006;10:21-29.

39 Forster A, Lambley R, Young JB: Is physical rehabilitation for older people in long-term care effective? Findings from a systematic review. Age Ageing 2010;39:169-175.

-40 Reisberg B, Ferris SH, de Leon MJ, Crook T: The global deterioration scale for assessment of primary degenerative dementia. Am J Psychiatry 1982;139:1136-1139.
Blankevoort/van Heuvelen/Boersma/ Luning/de Jong/Scherder 